\title{
Etiological Agents Isolated from Bronchoalveolar Lavage Samples in Patients with Lower Respiratory Tract Infections
}

\author{
K.N. Ravichitra* and U. Srinivasa Rao \\ Department of Microbiology, ASRAM Medical College, Eluru-534005, \\ West Godavari District, Andhra Pradesh, India \\ *Corresponding author
}

A B S T R A C T

\begin{tabular}{|l|}
\hline Ke y w o r d s \\
LRTI, Pneumonia, \\
BAL, bacteria, \\
fungus \\
\hline Article Info \\
\hline $\begin{array}{l}\text { Accepted: } \\
\text { 04 July } 2019 \\
\text { Available Online: } \\
\text { 10 August } 2019\end{array}$ \\
\hline
\end{tabular}

\section{Introduction}

Traditionally, the infections of respiratory system are classified as either upper or lower respiratory tract infections. ${ }^{1}$ Respiratory diseases have a wide spectrum extending from acute conditions such as pneumonias at one end to the chronic conditions such as obstructive lung diseases at the other end. ${ }^{2}$

Infection involving the lungs is termed pneumonia or lower respiratory tract infection and is a common clinical problem in the practice of respiratory care. The acute problems are often life -threatening but the burden is difficult to assess. ${ }^{2}$ Laboratory diagnosis of lower respiratory infections poses a number of major challenges. Diversity of etiological agents imposes diverse specimen requirements and laboratory approaches. ${ }^{3}$ The techniques used for sampling the tracheobronchial tree include the bronchial washing, bronchial brushing and BAL.

The specimens can be sent for various microbiological and cytological analyses. The more distal the sampling from the lower respiratory tract, the more specific it is.

BAL is a relatively safe and easy procedure, in which a lung segment is lavaged with sterile saline through the bronchoscope and recovered fluid can be used for various microbiological and cytological analysis. ${ }^{4}$ The 
advantage of BAL is that a wider area of the lung is sampled ${ }^{5}$ and that it is highly specific, but less sensitive. It has been studied extensively as a tool for diagnosing nosocomial pneumonia.

The relative frequencies and antimicrobial susceptibilities of the bacteria may vary considerably from one institution to another. Knowledge of which is very useful.

Hence this study was undertaken to know the rate of isolation of various bacteria from the Bronchoalveolar lavage samples in clinically suspected lower respiratory tract infections and the antimicrobial susceptibility pattern of the confirmed isolates, also to know the isolation rate of various fungi from those samples.

\section{Materials and Methods}

It is an observational study done in the Department of Microbiology, ASRAM. Bronchoalveolar lavage samples of clinically suspected lower respiratory infections received in the department for aerobic bacterial culture and sensitivity and for fungal examination during Jan 2014 and Dec 2016 were included in the study. Both inpatients and outpatients of both sexes and above 18 years of age were included in the study, those below 18 years of age were excluded from the study. BAL sample collected and transported immediately to the Microbiology department under aseptic conditions was processed.

After macroscopic examination, preliminary screening by gram staining, $\mathrm{KOH}$ and LPCB mounts was performed. Isolation of organisms was done by inoculating on the MacConkey, blood and chocolate agar using $4 \mathrm{~mm}$ calibrated inoculating loop and incubated for 18-24hrs. A small portion of the sample was also inoculated on SDA and incubated for 1week. ${ }^{6}$

\section{Processing for aerobic bacterial isolates}

All inoculated plates were observed for growth after the incubation period. A cut off value of $10^{3}$ to $10^{4}$ bacterial colonies per milli liter of BAL fluid was taken. ${ }^{7}$ Confirmation of the bacterial isolates was done by gram staining, hanging drop preparation, oxidase, catalase, coagulase and other biochemical tests.

\section{Antibiotic susceptibility testing}

Done on Muller-Hinton agar by Kirby-Bauer disc diffusion method. Commercially available antimicrobial impregnated antibiotic discs were used for sensitivity testing. Zone size was measured and reported according to CLSI guidelines (CLSI). ${ }^{8}$

\section{Processing of the fungal isolates}

SDA slants were observed for growth after one week and isolates confirmed by macroscopic and microscopic observation of LPCB mounts.

\section{Results and Discussion}

A total of 472 BAL samples that met the inclusion criteria were included in the study. Of the total samples $336(71.2 \%)$ were males and $136(28.8 \%)$ were female patients. Maximum number 223 (47.2\%) of samples were of the age group 40-60 years and minimum number of samples were of the age group 18-30 years. $383(81.1 \%)$ samples revealed growth of various bacterial organisms.89 (18.9\%) samples were culture negative. Culture positives in different LRTI are shown in the chart 1.

Majority 250(65.3\%) were Gram positive organisms whereas 133(34.7\%) were gram negative organisms. Various bacteria isolated from the samples are shown in the table 1. 
Chart.1 Culture positives in different LRTI

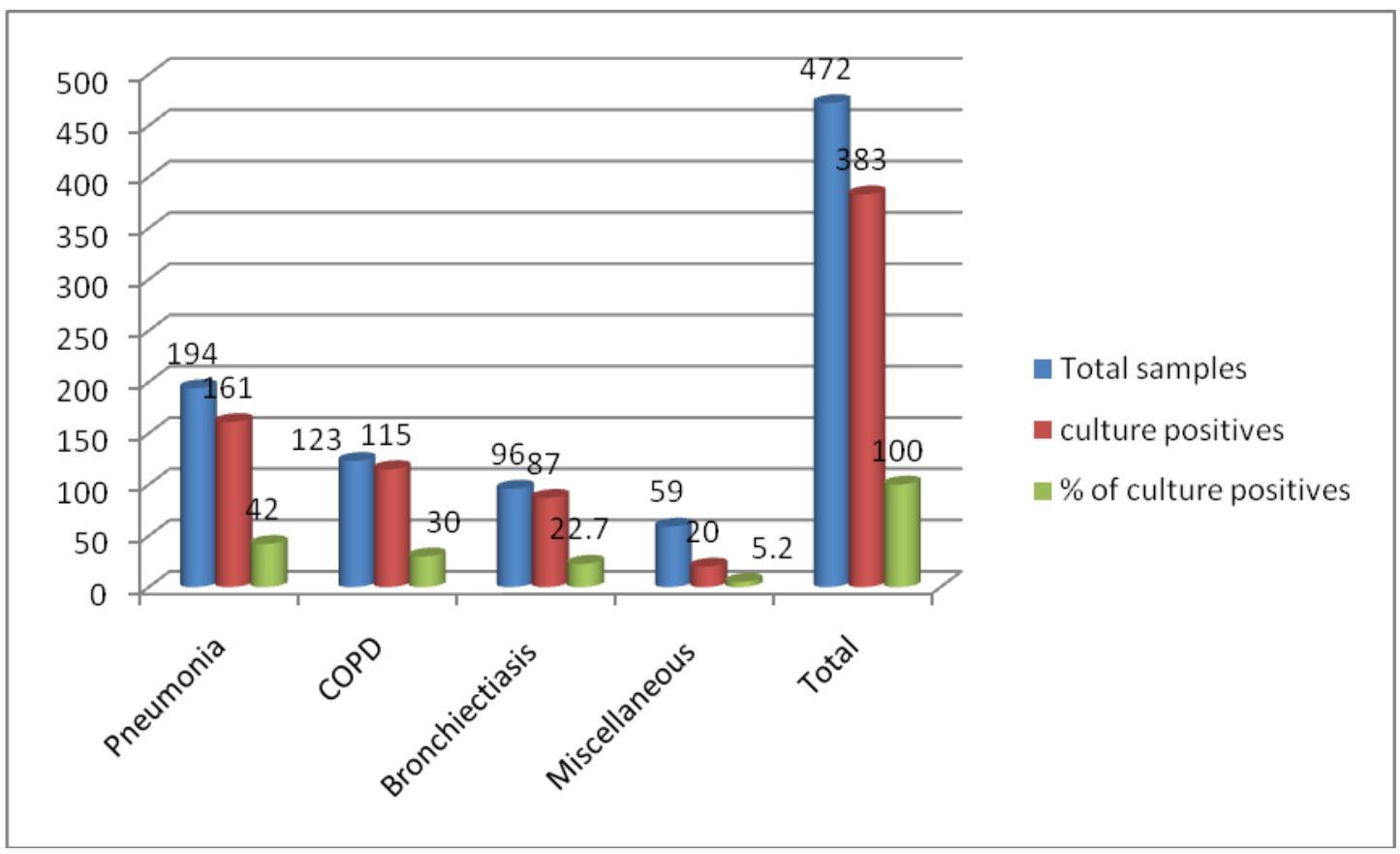

Chart.2 Fungal isolates from Bronchoalveolar lavage

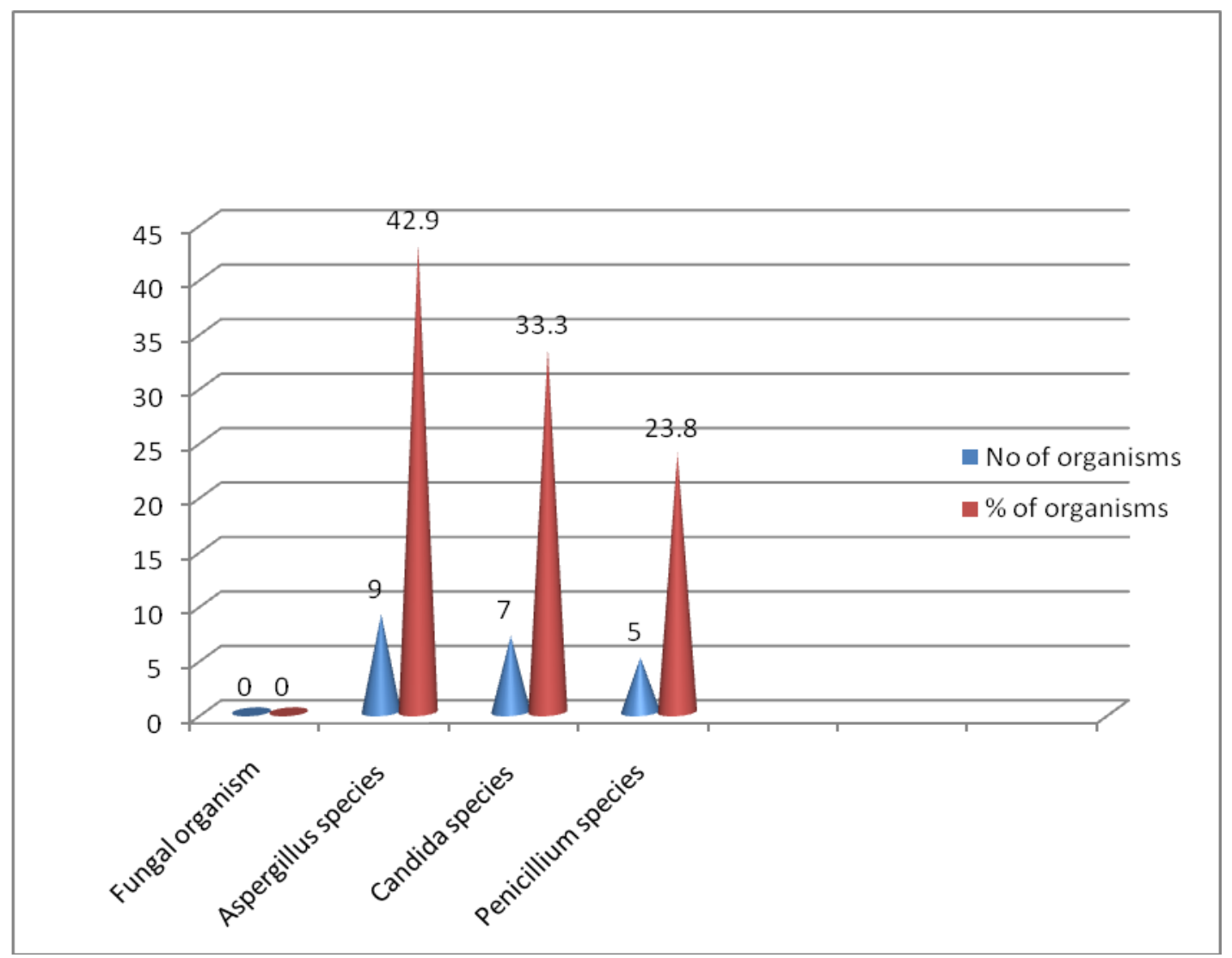


Table.1 Various bacterial organisms isolated from BAL

\begin{tabular}{|l|c|c|}
\hline Organisms & $\begin{array}{c}\text { No of organisms } \\
\text { isolated }\end{array}$ & $\begin{array}{c}\text { \% of organisms } \\
\text { isolated }\end{array}$ \\
\hline COPS & 131 & 34.2 \\
\hline S.pneumoniae & 80 & 20.9 \\
\hline Enterococcus & 39 & 10.2 \\
\hline Klebsiella pneumoniae & 71 & 18.5 \\
\hline Escherichia coli & 9 & 2.3 \\
\hline Pseudomonas & 53 & 13.8 \\
\hline Total & 383 & 100 \\
\hline
\end{tabular}

Gram positive organisms showed good sensitivity to Linezolid (99.2\%), piperacillin/tazobactum (94.4\%), moderate sensitivity to azithromycin (79.6\%), Ciprofloxacin (75.2\%) and Ceftraixone (83.6\%). Sensitivity to amoxicillin/clavulinic acid was only $(57.2 \%)$ in our study. Gram negative organisms showed maximum sensitivity to Imipenem (99.2\%) followed by piperacillin/tazobactum (89.5\%). Amikacin (78.2\%), Ciprofloxacin (60.9\%), Ceftiaxone $(74.4 \%)$ and amoxicillin/clavulinic acid (53.4\%). Of the 472 samples processed 21 (4.4\%) samples showed growth of various fungus. Isolation of various fungus is shown in chart 2. Aspergillus is the most common species isolated in our study followed by candida and Penicillium species.

LRTI 'S, especially pneumonia is the leading cause of infection related mortality. Our study showed that males constituted $72.1 \%$, which is similar to Eun Sun Kim et al., $72.9 \%{ }^{9}$ Culture positives in our study were $81.1 \%$ which is similar to the study conducted by Vivek et al., $89 \% .{ }^{10} 18.9 \%$ of samples in our study did not reveal any bacterial growth which is similar to the study conducted by Rasmussen et al., 24\%. ${ }^{11}$ Gram positive cocci predominated in our study $65.3 \%$, whereas study conducted by Eun Sun Kim et al., showed $28 \%$ isolation of gram positive cocci. ${ }^{9}$ $18.5 \%$ of the isolates in our study were Klebsiella pneumoniae which is similar to the study conducted by Rahul Magazine et al., $19.4 \%$. ${ }^{12}$ Our study showed $2.3 \%$ of isolates were Escherichia coli, whereas Rahul Magazine et al., reported $5.1 \%,{ }^{12}$ higher than that reported by us. Fungal growth was noted in $4.4 \%$ of our samples whereas Bhatia et al., reported $12 \%$, slightly higher than that reported by us. ${ }^{13}$ Sripriya et al., reported $29.9 \%$ isolation of fungus, ${ }^{14}$ much higher than that reported by us. $42.9 \%$ of fungal isolates in our study were of Aspergillus species whereas H. Levy et al., reported 24.4\% isolation of Aspergillus species lower than that reported by us. ${ }^{15}$

Bronchoalveolar lavage is an important adjunct for the early diagnosis of lower respiratory tract infections. This diagnostic test helps the clinician to start early specific treatment and thus reduce the morbidity and mortality.

\section{References}

1. Ioannis Mastoris., Sotirios Tsiodras. 2015. Textbook of Respiratory and Critical Care infections: 1st edition, 102-103.

2. R. Narasimhan., AR Gayathri. 2017. Text book of Pulmonary and Critical care medicine: $2^{\text {nd }}$ edition, $385-386$.

3. Bobbi S. Pritt., Joseph D.C. 2010. Murray \& Nadel's Textbook of Respiratory Medicine: $5^{\text {th }}$ edition, 368. 
4. Steven K Schmitt., David L. Long worm. 2013. Egan's Fundamentals of Respiratory Care: 10th edition, 506.

5. Mark A Woodhead.2008.Clinical Respiratory Medicine: 3rd edition, 331.

6. J. Gerald Collee., Barrie P. Marmion et al., 2012. Mackie \& Mc Cartney: Practical Medical Microbiology: 14th edition, 413-418.

7. Patricia M. Tille. 2014. Bailey \& Scott s Diagnostic Microbiology: 13th edition, 887.

8. Franklin R. Cockerill. 2012. CLSI Performance Standards for Antimicrobial Disk Susceptibility Tests; Approved Standard: Eleventh Edition.

9. Eun Sun Kim., Eui-Chong Kim et al., 2012. Bacterial Yield from Quantitative Cultures of Bronchoalveolar Lavage Fluid in Patients with Pneumonia on Antimicrobial Therapy. Korean J Intern Med. 27 (2): 156-162.

10. Vivek KU, Nutan Kumar DM et al., 2016. Microbiological profile of bronchoalveolar lavage fluid in patients with chronic respiratory diseases: a tertiary care hospital study. Int J Med Res Rev. 4(3): 330-337.
11. T. R. Rasmussen., J. Korsgaard et al., 2001. Quantitative culture of bronchoalveolar lavage fluid in community-acquired lower respiratory tract infections. Respiratory Medicine. 95: 885-890.

12. Rahul Magazine., Bharti Chogtu et al., 2013. Bacterial isolates from the bronchoalveolar lavage fluid of patients with pneumonia not responding to initial antimicrobial therapy. Sahel Medical Journal. 16(3): 102-106.

13. G. C. Bhatia., N. Hemvani et al., 2006. Role of bronchioalviolar lavage in pulmonary infections. Lung India. 23: $147-150$.

14. Sripriya, C.S., S. Thasneem Banu et al., 2017. Mycological Profile of Bronchial Wash Specimens in Patients with Lower Respiratory Tract Infections. Int.J.Curr.Microbiol.App.Sci. $\quad 6(11)$ : 176-182.

15. H. Levy, D., A. Horak et al., 1992. The value of Bronchoalveolar lavage and bronchial washings in the diagnosis of invasive pulmonary aspergillosis. Respiratory Medicine. 86: 243-248.

\section{How to cite this article:}

Ravichitra, K.N. and Srinivasa Rao, U. 2019. Etiological Agents Isolated from Bronchoalveolar Lavage Samples in Patients with Lower Respiratory Tract Infections. Int.J.Curr.Microbiol.App.Sci. 8(08): 1-5. doi: https://doi.org/10.20546/ijcmas.2019.808.001 